\title{
Bio-Inspired Routing Protocol Based on Pheromone Diffusion in Mobile Ad Hoc Networks
}

\author{
Hyun-Ho Choi \\ Dept. of Electrical, Electronic \\ and Control Engineering \\ Hankyong National University \\ Anseong 17579, Korea \\ hhchoi@hknu.ac.kr
}

\author{
Jung-Ryun Lee \\ School of the Electrical \\ Engineering \\ Chung-Ang University \\ Seoul 06974, Korea \\ jrlee@cau.ac.kr
}

\author{
Bongsoo Roh, Mijeong \\ Hoh, HyungSeok Choi \\ Agency for Defense \\ Development \\ Daejeon 34188, Korea \\ \{saintroh,hmj,chs\}@add.re.kr
}

\begin{abstract}
Bio-inspired routing protocols use the principle of swarm intelligence, which finds the optimal path to the destination in a distributed and autonomous way in dynamically changing environments; therefore, they can maximize the routing performance, reduce the control overhead, and recover a path failure quickly according to the change in the network topology. In this paper, we propose a bio-inspired routing protocol for mobile ad hoc networks. The proposed protocol uses a technique of overhearing for obtaining routing information without additional overhead. Through overhearing, a pheromone is diffused around the shortest path between the source and the destination. On the basis of this diffused pheromone, a probabilistic path exploration is executed and the useful alternative routes between the source and the destination are collected. Therefore, the proposed routing protocol can gather up-to-date effective routing information while reducing the control overhead. The simulation results show that the proposed routing protocol outperforms the typical ad hoc on-demand distance vector (AODV) and AntHocNet protocols in terms of the delivery ratio and the end-to-end delay and significantly decreases the routing overhead against AntHocNet.
\end{abstract}

\section{Categories and Subject Descriptors}

C.2.2 [Network Protocols]: Routing protocols

\section{General Terms}

Algorithm, Design, Performance

\section{Keywords}

Routing protocol, bio-inspired routing, mobile ad hoc network, overhearing, pheromone diffusion

\section{INTRODUCTION}

Mobile ad hoc networks (MANETs) have a dynamic topology and an unstable wireless channel. Link failure frequently occurs because of path loss, fading, interference, limited radio resource, and node mobility. Node failure may happen because of limited battery power and breakdown or demolition of user terminals. Moreover, there exist multiple multihop sessions and considerable data traffic that have various quality of service (QoS) requirements in MANETs. Therefore, a MANET provides a highly dynamic topology for the routing protocol.

In the dynamic topology environment, the topology information identified by a routing protocol is already past information at the time of data transmission. Therefore, it is impossible for nodes to transfer data through the optimal path all the time in MANETs, but it is just possible to use the best available path by using the currently identified topology information. Considering this realistic restriction, one of the key factors to determine the performance of the routing protocol is the amount of the latest useful routing information that can be acquired while reducing the overhead of the control packet transmission. Inherently, there exists a tradeoff between the control overhead and the routing performance; hence, the routing protocol should be designed to minimize the overhead while adaptively satisfying the required performance for a given network environment [1].

Various types of routing protocols have been proposed for MANETs [2], [3]. They have different characteristics according to the design philosophy. A proactive routing protocol generates control packets periodically and thus, induces a high routing overhead. On the other hand, a reactive routing protocol is event-driven and thus causes a long path setup delay. In addition, a single path routing protocol is vulnerable to topology changes. On the other hand, a multipath routing protocol consumes more network capacity and terminal resources. Moreover, a source routing protocol creates additional overhead per data packet to include the complete path to the destination in the header. However, a hop-byhop routing protocol requires every node to manage its own routing table.

Compared with the typical MANET routing protocols, the bio-inspired routing protocols have the following advantages: First, a routing protocol inspired by the swarm behavior is 
suitable for autonomously finding the shortest path to the destination in MANETs because a swarm of ants or bees finds the optimal route to food in a distributed manner in dynamic environments. Second, the bio-inspired routing protocol can efficiently maintain the shortest path while reducing the occurrence of the overhead because the swarm intelligence maintains the optimal path flexibly in a resourcelimited environment. Third, the bio-inspired routing protocol can quickly repair a link or node failure because the swarm effectively solves various unpredictable problems in the real world [4]-[6].

In this paper, we propose a bio-inspired routing protocol for MANETs. The proposed routing protocol uses a function of overhearing from the broadcast nature of wireless media in order to obtain the routing information without any overhead. Through this overhearing technique, a pheromone is diffused around the shortest path between the source and the destination. Thereafter, based on this diffused pheromone, a probabilistic path exploration is executed around the shortest path and the alternative routes that are disjoint from the shortest path are discovered. These operations are inspired by the behavior of ants [7], [8]. That is, the ants' continuous movement between the source and the destination accumulates the pheromones on the shortest path and then these pheromones naturally diffuse into the periphery of this path. Once the shortest path in use is broken, the ants try to find another route along the diffused pheromone around the shortest path. By applying such a navigation method of real ants, the proposed routing protocol can achieve an efficient and seamless packet transmission while reducing the control overhead. The proposed routing protocol is validated by an OPNET simulator with respect to the end-to-end performance and the control overhead.

The rest of this paper is organized as follows: Section 2 reviews the operations of ad hoc on-demand distance vector (AODV) and AntHocNet as the benchmark protocols of the proposed routing protocol. Section 3 explains the operation of the proposed bio-inspired routing protocol in detail. Section 4 shows the performance of the proposed protocol in terms of the routing performance and the control overhead compared with the AODV and AntHocNet protocols. Finally, Section 5 presents the conclusions drawn in this study.

\section{RELATED WORKS}

For the most important benchmark protocol, we choose AODV and AntHocNet. AODV is a representative MANET routing protocol and AntHocNet is a representative bio-inspired routing protocol. In this section, we describe the characteristics of AODV and AntHocNet and review their limitations.

\subsection{AODV}

AODV is by far the most cited and studied routing protocol for MANETs [9], [10]. AODV is the on-demand version of the destination-sequenced distance vector (DSDV) protocol [11]. Like DSDV, it uses hop-by-hop routing based on distance vector tables, but does not use the Bellman-Ford algorithm. Being a reactive algorithm, AODV starts a route discovery process when a node has data to send to a destination that it has no information about. In this process, the source node floods a route request (RREQ) over the network. During this flooding process, the intermediate nodes receiving the RREQ leave a pointer towards the source in their routing table. When the RREQ reaches the destination, a route reply (RREP) is sent back to the source, which follows the preset pointers back to the source and adds the reverse pointers towards the destination. By doing so, when the RREP arrives at the source, the path between the source and the destination is newly established. Then, data packets are routed from the source to the destination following the pointers set by the RREP. When there is a failure anywhere along the path, a route error (RERR) is generated to warn the source, which starts a new route discovery process.

\subsection{AntHocNet}

AntHocNet is a routing protocol based on the distance vector but uses the pheromone instead of it [12]-[14]. The pheromone is a stochastic value derived from the distance vector, and its use is a key feature of the bio-inspired routing protocols. AntHocNet is a hybrid routing protocol, consisting of the reactive route setup and the proactive route maintenance processes. AntHocNet starts a reactive route setup process when the source has no routing information about the destination of the data packet. The source floods a reactive forward ant over the network to find a route to the destination. Each intermediate node forwards the reactive forward ant via unicasting in case it has routing information about the ant's destination, and via broadcasting otherwise. Unlike AODV, the reactive forward ants store the full array of nodes that they have visited on their way to the destination. The first reactive forward ant that reaches the destination is converted into a reactive backward ant, which retraces the exact path that was followed by the forward ant back to the source. On its way, each intermediate node and the source update the routing tables on the basis of the quality information collected by the reactive backward ant; therefore, the first path between the source and the destination is established.

Once the first route is constructed by the reactive route setup process, AntHocNet starts the proactive route maintenance process, which consists of two subprocesses: pheromone diffusion and proactive ant sampling. The aim of the pheromone diffusion subprocess is to spread the pheromone information that was placed by the ants. All nodes in the network periodically broadcast hello messages containing the best pheromone information that they have available. Upon receiving the hello messages, neighboring nodes derive a new pheromone for themselves and further forward it in their own periodic broadcasts. The pheromone obtained by this pheromone diffusion is called a virtual pheromone and is managed separately from the regular pheromone placed by the ants.

The virtual pheromone is used for supporting the proactive ant sampling. In this subprocess, the source node periodically sends out proactive forward ants towards the destination of the session. These ants construct a path in a stochastic way, choosing a new next hop probabilistically at each intermediate node. When calculating the probability of taking the next hop, proactive forward ants consider the maximum between the regular and the virtual pheromones, 
as follows:

$$
P_{i n}^{d}=\frac{\left[\max \left(\tau_{i n}^{d}, \omega_{i n}^{d}\right)\right]^{\beta_{2}}}{\sum_{j \in \mathbf{N}_{i}^{d}}\left[\max \left(\tau_{i j}^{d}, \omega_{i j}^{d}\right)\right]^{\beta_{2}}}, \quad \beta_{2} \geq 1
$$

where $P_{i n}^{d}$ denotes a probability that a node $i$ chooses node $n$ as the next hop to reach the destination $d, \tau_{i j}^{d}$ and $\omega_{i j}^{d}$ represent the regular and the virtual pheromones, respectively, for the route from $i$ to $d$ over $j, \mathbf{N}_{i}^{d}$ denotes the set of neighbors of $i$ over which a path to $d$ is known, and $\beta_{2}$ represents a parameter that controls the exploratory character of the ants. In this way, the proactive forward ants can leave the routes that were followed by previous ants and follow the other routes that have emerged from pheromone diffusion. Once a proactive forward ant reaches the destination, it is converted into a proactive backward ant that travels back to the source and leaves the regular pheromone along the way. That is, proactive ants can follow a virtual pheromone and then, once they have experienced that it leads to the destination, convert it into a regular pheromone. One could say that pheromone diffusion suggests new paths and that proactive ants check them out.

In AntHocNet, data packets are forwarded by using only regular pheromones. Each forwarding decision is taken using a stochastic formula, as follows:

$$
P_{i n}^{d}=\frac{\left(\tau_{i n}^{d}\right)^{\beta_{3}}}{\sum_{j \in \mathbf{N}_{i}^{d}}\left(\tau_{i j}^{d}\right)^{\beta_{3}}}, \quad \beta_{3} \geq 1
$$

which gives preference to the next hops that are associated with higher pheromone values.

\subsection{Limitations of Typical Routing Protocols}

The flooding mechanism for route discovery in AODV induces a large overhead because it propagates the RREQ to the entire network. Moreover, it should perform the flooding every time the link failure occurs because AODV is a singlepath routing protocol. Eventually, the flooding overhead becomes more serious in the relatively high mobility environment. In addition, AODV does not use any route maintenance process after the route setup and thus, has no additional overhead for it. However, it has a disadvantage that nodes cannot always use the optimal path if the topology changes. In order to overcome this problem, some AODVbased multipath routing protocols have been proposed in the literature, but they require a high operational complexity and have not been formally discussed in the standardization [15]-[17].

AntHocNet generates a large overhead in the proactive route maintenance process because all the nodes in a network must periodically transmit hello messages including the best pheromone information for the pheromone diffusion, and the proactive ant packets must be periodically exchanged between the source and the destination for the proactive ant sampling. This periodic transmission of control packets in each node acts as a large overhead and thus, lowers the transmission efficiency of the data packets in MANETs. Furthermore, the pheromone diffusion in AntHocNet basically follows the routing table update mechanism of DSDV, but it is not suitable for highly dynamic or large-scale networks [11]. Therefore, the pheromone diffusion process in AntHoc-
Net decreases the convergence speed to adapt to topology changes in a high mobility environment; thus, it may not provide accurate routing information for the destination. Moreover, the proactive ant sampling in AntHocNet uses the maximum between the regular and the virtual pheromones to calculate the probability of the next hop. Thus, if there is no significant change in the virtual pheromone, it mainly depends on the regular pheromone and leads to a problem called stagnation, where the ants explore the same path repeatedly. The stagnation problem implies the unnecessary generation of proactive ants; therefore, the overhead is increased and the probability to select the optimal path is consequently decreased according to the topology changes [14].

\section{PROPOSED BIO-INSPIRED ROUTING PRO- TOCOL}

In this section, we provide an overview of the proposed bioinspired routing protocol and then explain its operation in detail.

\subsection{Overview}

Figure 1 shows seven snapshots to explain the overall operation of the proposed routing protocol. In each snapshot, the following operations are executed:

(a) Route Setup: The shortest path from the source to the destination is set up at the beginning. The proposed routing protocol uses the same route setup process as AODV for a fast route setup. After the route setup, data and acknowledgement packets and forward and backward exploitation ants are transferred along the found shortest path. The forward and backward exploitation ants are newly defined agent packets for the proposed routing protocol and are transmitted periodically in order to maintain the shortest path between the source and the destination. The details of the exploitation ants will be provided in Section 3.2.

(b) Overhearing: Nodes in the vicinity of the shortest path overhear the forward exploitation ant transmitted from the source to the destination and/or the backward exploitation ant transmitted from the destination to the source. Only the node that overhears both the forward and backward exploitation ants within a certain period of time becomes a one-hop neighbor of that path.

(c) Pheromone Diffusion: One-hop neighbors update their routing table information, such as cost, update time, and pheromone, by using the overheard forward and backward exploitation ants. In addition, the one-hop neighbors include the smallest cost value about the destination of the overheard source-destination pair in a hello message and periodically broadcast it. If the adjacent nodes receive this hello message, they update the cost and pheromone values for that destination. In this way, the pheromone is diffused locally by not only the overhearing but also the broadcast of hello messages by one-hop neighbors. All the pheromone information obtained by this pheromone diffusion process is set to an inactive pheromone because such a pheromone is not directly identified by ant packets between the source and the destination. 


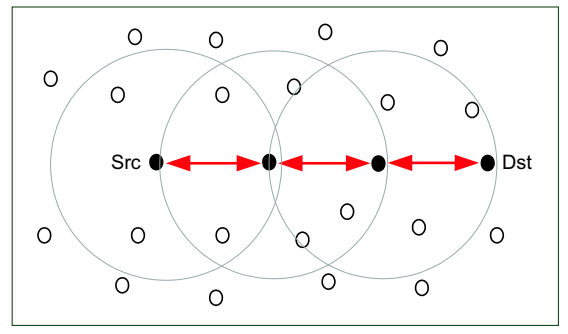

(a) Route Setup

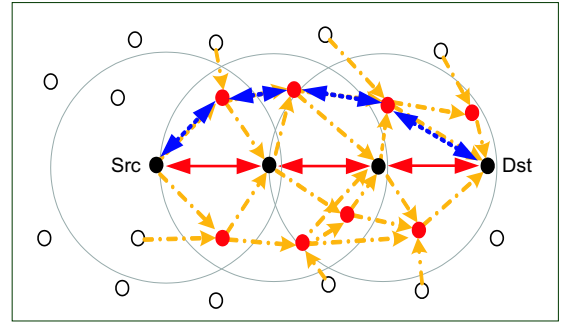

(d) Route Maintenance I

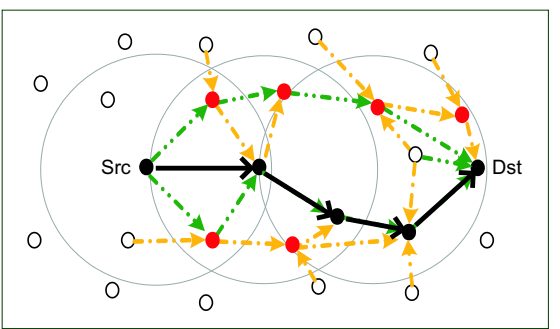

(g) Data Forwarding

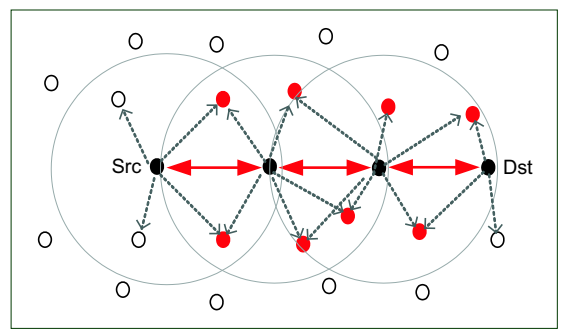

(b) Overhearing

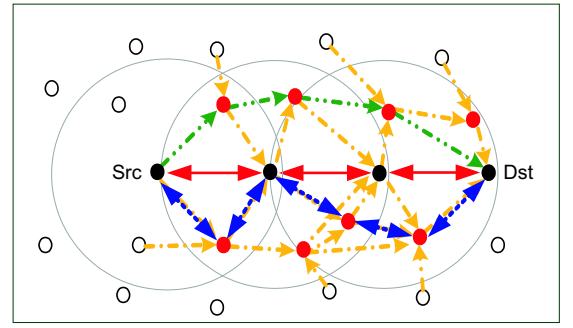

(e) Route Maintenance II

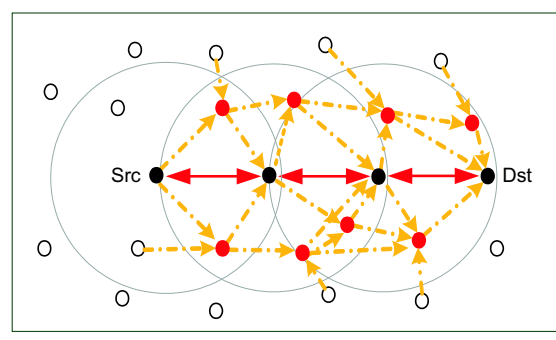

(c) Pheromone Diffusion

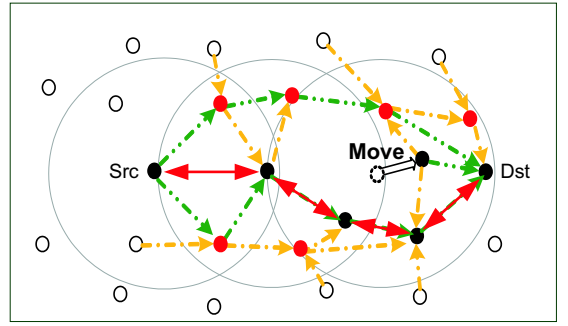

(f) Route Maintenance III

Figure 1: Operation of the proposed bio-inspired routing protocol.

(d)-(f) Route Maintenance: For route maintenance, the source node periodically generates forward exploitation ants and forward exploration ants. If the destination node receives a forward exploitation ant and a forward exploration ant, it transmits to the source node the backward exploitation ant and the backward exploration ant, respectively, as a response. The forward/backward exploitation ant moves to the destination/source along the highest active pheromone in order to exploit the current best path between the source and the destination, which leads to the maintenance of the shortest path and the determination of the one-hop neighbors around it. On the other hand, the forward exploration ant moves to the destination probabilistically in proportion to the inactive pheromone diffused around the exploitation ant path in order to offer alternative routes. The inactive pheromone on the path passed by the exploration ant is changed into the active pheromone by the backward exploration ant, and then, this path can be used for transmitting the data and ACK packets and the exploitation ant packets. On the other hand, the active pheromone is changed into the inactive pheromone after a certain period of time from the update time. Figures 1(d) and 1(e) show that some alternative paths are set around the shortest path between the source and the destination by the periodic transmission of the exploration ant. As shown in Fig- ure 1(f), the exploitation ant uses a new shortest path if the topology is changed by the movement of a node. In the dynamic topology, the exploitation ants try to ensure the shortest path between the source and the destination and the exploration ants try to provide the alternative paths around this shortest path.

(g) Data Forwarding: Data packets are forwarded to the destination only along the active pheromones. The next hop is determined probabilistically in proportion to the active pheromone.

\subsection{Detailed Operation}

We explain the detailed operation of the proposed bio-inspired routing protocol according to the following seven key functions:

\subsubsection{Routing Table Management}

Basically, the routing table records the multiple path information for one destination. In addition to the typical information in the existing routing table, the update time, pheromone, and status are managed additionally in the proposed routing protocol [10]. Figure 2 illustrates an example of a routing table that manages the information for the destination node $d$. The role of each piece of information is as follows: 


\begin{tabular}{|c|c|c|c|c|c|c|}
\hline \multirow{2}{*}{ Dst } & & \multicolumn{5}{|c|}{ Next node that is selected by forward exploitation ant or data packet } \\
\hline & Next & Cost & Seq. Num. & Update Time & Pheromone & Status \\
\hline \multirow{6}{*}{$d$} & a & 4 & 10 & $09: 29: 45$ & 0.33 & active \\
\hline & b & 6 & 11 & 09:30:00 & 0.28 & active \\
\hline & c & 7 & 9 & 09:28:20 & 0.14 & active \\
\hline & $x$ & 5 & $\mathrm{n} / \mathrm{a}$ & 09:29:00 & 0.21 & inactive \\
\hline & $\mathrm{y}$ & 9 & $\mathrm{n} / \mathrm{a}$ & 09:28:30 & 0.11 & inactive \\
\hline & z & 10 & $\mathrm{n} / \mathrm{a}$ & $09: 26: 15$ & 0.005 & inactive \\
\hline
\end{tabular}

Figure 2: Example of routing table in the proposed routing protocol.

- Next: This indicates the next node to which the packet can be forwarded. Since the proposed routing protocol is the multipath routing protocol, the multiple next nodes for one destination are managed.

- Cost: This implies the total cost consumed to transmit a packet to the destination over the next node. The cost information is derived from the RREQ, RREP, exploitation ant, and exploration ant packets. The cost can be defined using various metrics, such as the number of hops, delay, expected transmission time (ETT), expected transmission count (ETX), received signal strength (RSS), and bandwidth [3].

- Sequence Number: This is updated as the sequence number in the received control packet only if the received sequence number is greater than the recorded sequence number. The sequence number is used for preventing the duplicated update from the same control packet.

- Update Time: This registers the time when the corresponding routing information is updated.

- Pheromone: This indicates the goodness of the next node to deliver a packet to the destination. The pheromone is inversely proportional to the elapsed time as well as the cost. That is, the pheromone decreases as the elapsed time increases by considering the evaporation of the pheromone. Here, the elapsed time $t$ is defined as " $t=$ current_time - update_time". Therefore, the pheromone $\tau_{i j}^{d}$ for the route from a node $i$ over the next node $j$ to reach the destination $d$ is defined as $\tau_{i j}^{d}=\left(1 / c_{i j}^{d}\right)^{\alpha}\left(1 / t_{i j}^{d}\right)^{\beta}$, where $c_{i j}^{d}$ and $t_{i j}^{d}$ denote the cost and the elapsed time for the corresponding route, respectively, and $\alpha \geq 1$ and $\beta \geq 1$ represent the weighting parameters.

- Status: The status is set to "active" when the route information is obtained by receiving the control packets (i.e., RREQ, RREP, RERR, and ants). However, it is set to "inactive" when the route information is obtained by the pheromone diffusion (i.e., overhearing the exploitation ants or receiving the hello message). The active status is changed into the inactive status after the ROUTE_TIMEOUT timer expires, and the inactive route information is deleted after the ROUTE_DELETE timer expires. The active route information is used for forwarding the data and exploitation ants, and the inactive route information is used for forwarding the exploration ant.

For the example shown in Figure 2, the data packet and the forward exploitation ant destined for $d$ are forwarded to $a$ because its pheromone is the highest among the active route information. On the other hand, the forward exploration ant is likely to be forwarded to $x$ with a high probability in proportion to the pheromone values in the inactive route information. The details about this selection algorithm are provided in Section 3.2.5.

\subsubsection{Route Setup}

The route setup procedure starts when there is no routing information about the destination of the data packet generated at a node. The basic route setup procedure follows the same flooding mechanism as AODV for the fast route setup. Therefore, the source floods the RREQ over the network and the destination replies to the source with the RREP when receiving the RREQ. If the RREP arrives at the source, the routing path between the source and the destination is established. If the source does not receive the RREP until the RREQ timeout occurs, it retries the RREQ flooding.

\subsubsection{Overhearing}

Every node in the network overhears the forward and backward exploitation ants that are not intended for it. Through this overhearing process, the node can determine whether or not it is a one-hop neighbor of a certain source-destination path by itself. The node becomes the one-hop neighbor only when it overhears both the forward and the backward exploitation ants for the same source-destination pair during a predetermined time. The one-hop neighbors calculate the cost to the destination from the overheard ant packets and update the routing table. The updated cost value is calculated as $c_{i j}^{d}=c_{j}^{d}+c_{i}^{j}$. That is, the total cost of the route from $i$ to $d$ over $j, c_{i j}^{d}$, is the sum of the cost from $j$ to $d$, $c_{j}^{d}$, which is contained in the overheard ant packets, and the cost from $i$ to $j, c_{i}^{j}$, which is locally maintained by the hello messages.

\subsubsection{Pheromone Diffusion}

The first pheromone diffusion is executed by the overhearing process. The second is achieved by the one-hop neighbors' periodic broadcast of the hello messages that contains the smallest cost for the destination of the overheard sourcedestination pair. If some nodes receive the hello messages from the one-hop neighbors, they update the cost to the corresponding destination by using $c_{i j}^{d}=s_{j}^{d}+c_{i}^{j}$ where $s_{j}^{d}$ denotes the smallest cost from $j$ to $d$ obtained from the hello message and $c_{i}^{j}$ represents the cost from $i$ to $j$ maintained locally. With this cost update, the additional routing information is updated. These pheromones are treated as inactive pheromones because they are indirectly obtained by overhearing the exploitation ants and broadcasting the hello messages.

\subsubsection{Route Maintenance}

The maintenance of the shortest path is performed by the exploitation ants and the search for alternative paths is 
performed by the exploration ants. The source periodically transmits the forward exploitation ant after the initial route setup. The forward exploitation ant selects the next hop with the highest pheromone in the active routing information and is forwarded to the destination via unicasting. Upon receiving the forward exploitation ant, the destination generates the backward exploitation ant as a response. In the same way, the backward exploitation ant is forwarded to the next node with the highest pheromone toward the source via unicasting. During this process, the nodes on the path through which the forward and backward exploitation ants have passed update their routing table for the source and the destination nodes. In this way, the shortest path is maintained because the exploitation ants move along with the best active pheromone between the source and the destination.

Moreover, the source periodically transmits the forward exploration ant after receiving the initial backward exploitation ant. The forward exploration ant is forwarded via unicast to the next node, which is selected probabilistically in proportion to the inactive pheromones, by using the following equation:

$$
P_{i n}^{d}=\frac{\left(\mu_{i n}^{d}\right)^{\gamma_{1}}}{\sum_{j \in \mathbf{N}_{i}^{d}}\left(\mu_{i j}^{d}\right)^{\gamma_{1}}}, \quad \gamma_{1} \geq 1
$$

where $P_{i n}^{d}$ denotes the probability that node $i$ chooses node $n$ as the next hop for destination $d, \mu_{i j}^{d}$ represents the inactive pheromone for the route from $i$ over $j$ to reach $d$, $\mathbf{N}_{i}^{d}$ indicates the set of neighbors of $i$ over which an inactive pheromone is known, and $\gamma_{1}$ denotes a parameter value that can control the exploratory behavior of the ants. Unlike the exploitation ant, the forward exploration ant records the information of the intermediate nodes, through which it arrives at the destination, in its header. Upon receiving the forward exploration ant, the destination generates the backward exploration ant as a response. The backward exploration ant is delivered to the source exactly along the reverse of the path through which the forward exploration ant has passed. In this process, the nodes on the path through which the forward and the backward exploitation ants have travelled update their routing table and set the status to "active". That is, the inactive pheromones are changed into active pheromones by the exchange of exploration ants, and then, these active pheromones can be used for forwarding the exploitation ants and data packets.

\subsubsection{Data Forwarding}

For the load balancing in the network, data packets are forwarded probabilistically on the basis of the following probability:

$$
P_{i n}^{d}=\frac{\left(\tau_{i n}^{d}\right)^{\gamma_{2}}}{\sum_{j \in \mathbf{N}_{i}^{d}}\left(\tau_{i j}^{d}\right)^{\gamma_{2}}}, \quad \gamma_{2} \geq 1
$$

where $\tau_{i j}^{d}$ denotes the active pheromone for the route from $i$ over $j$ to reach $d, \mathbf{N}_{i}^{d}$ represents the set of neighbors of $i$ over which an active pheromone is known, and $\gamma_{2}$ indicates a parameter value that controls the spreading of data. This formula is very similar to the one used for the forward exploration ant, as shown in (3), but uses the active pheromone and a different parameter $\gamma_{2}$. This separates the exploita-
Table 1: Simulation parameters

\begin{tabular}{|l|l|}
\hline \multicolumn{1}{|c|}{ Name } & \multicolumn{1}{c|}{ Value } \\
\hline Number of simulated topologies & 40 \\
\hline Simulation time & $900 \mathrm{~s}$ \\
\hline Number of nodes $(N)$ & 100 \\
\hline Width = Height & $1000 \mathrm{~m}$ \\
\hline Communication range $\left(=\frac{2 \times \text { width }}{\sqrt{N}}\right)$ & $200 \mathrm{~m}$ \\
\hline Mobility model & Random waypoint \\
\hline Speed interval & Rand $(0,5 \sim 40) \mathrm{m} / \mathrm{s}$ \\
\hline Pause interval & Rand $(0,30) \mathrm{s}$ \\
\hline ROUTE_TIMEOUT timer & $3 \mathrm{~s}$ \\
\hline ROUTE_DELETE timer & $3 \mathrm{~s}$ \\
\hline Period of data packet generation & $0.25 \mathrm{~s}$ \\
\hline Period of hello message generation & $1 \mathrm{~s}$ \\
\hline Period of proactive forward ant gen. & $1 \mathrm{~s}$ \\
\hline Period of forward exploitation ant gen. & $1 \mathrm{~s}$ \\
\hline Period of forward exploration ant gen. & $1 \mathrm{~s}$ \\
\hline TTL of forwarding exploration ant & 20 \\
\hline Weight constants & $\alpha=1, \beta=1$ \\
\hline Exploratory parameter & $\gamma_{1}=1, \gamma_{2}=20$ \\
\hline
\end{tabular}

tion path and the exploration path between the source and the destination.

By the adaptation of $\gamma_{2}$, the data forwarding is spread less or more with respect to the best available routes. By setting $\gamma_{2}$ low, the data are spread over multiple routes, and thus, the traffic load to the network is distributed. By setting $\gamma_{2}$ high, on the other hand, the data are concentrated on the best routes, and thus, the shortest end-to-end delay is achieved [14].

\subsubsection{Route Recovery}

Link failures are detected if lower-layer protocols report the failure of the unicast transmission of a control or data packet, or if a node fails to receive the periodic hello messages from its neighbors. If a node has no next hop information for data transmission because of a link failure, it deletes the corresponding routing table information and broadcasts an RERR to notify its neighbors of the link failure. The nodes receiving the RERR update their routing table for the link to the node that sent this RERR. If some nodes still have data to send, but no next hop information in the updated routing table, they rebroadcast the RERR. In this way, the link failure is notified to the relevant nodes on and on. Eventually, if the source receives the RERR and has no next hop information for the destination, it restarts the route setup process.

\section{RESULTS AND DISCUSSION}

An OPNET simulator is used for the performance evaluation [18]. Table 1 summarizes the parameters used for the simulation. To concentrate the effect of the routing process, we consider only the topology in which the initial number of hops between the source and the destination is more than three [19]. Moreover, we do not consider the expanding ring search in AODV and assume that the RREP is replied only at the destination [20]. We also assume a unidirectional UDP traffic that does not require the ACK [14]. 


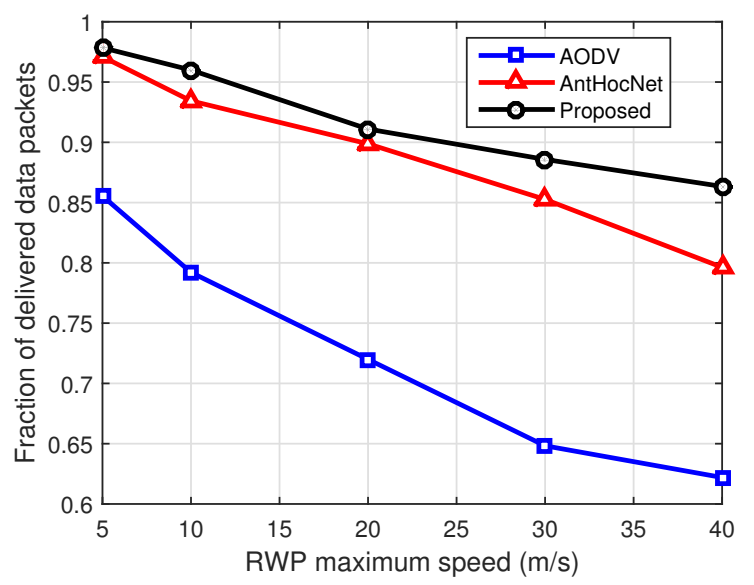

Figure 3: Fraction of delivered data packets vs. RWP maximum speed.

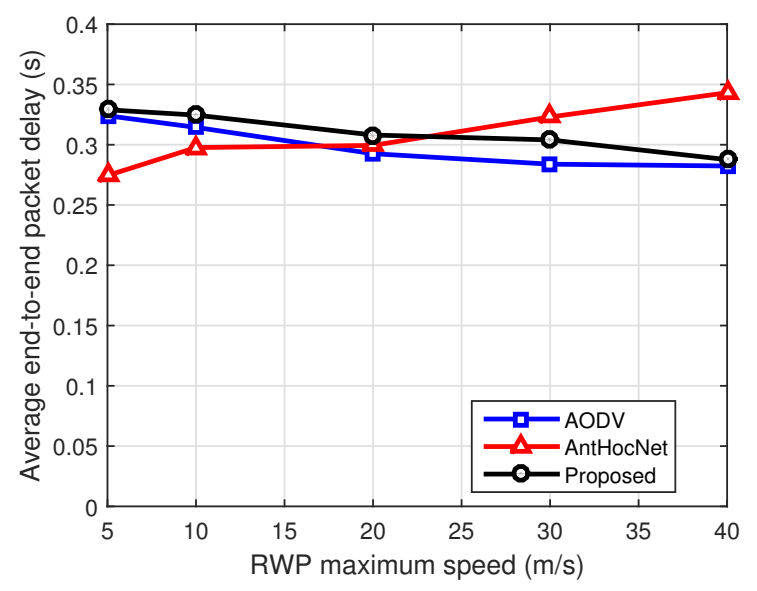

Figure 4: Average end-to-end packet delay vs. RWP maximum speed.

Figure 3 shows the fraction of correctly delivered data packets versus the maximum node speed for random waypoint (RWP) mobility. Data packets can be lost because of a link failure because the retransmission of lost data is not allowed by assuming the UDP traffic. Overall, the data delivery ratio decreases as the mobility increases because link failures occur more frequently. AODV has the lowest delivery ratio because it is not a multipath routing protocol. Compared with AODV, both AntHocNet and the proposed protocol show high delivery ratios. However, the proposed protocol has a considerably higher delivery ratio than AntHocNet when the mobility increases. This is because the proposed routing protocol finds more effective alternative paths than AntHocNet in order to prepare for a future link failure.

Figure 4 shows the average end-to-end delay of the delivered data packets versus the maximum node speed for RWP mobility. As the mobility increases in AODV, the link failures occur more often and the shortest path is re-established, which has a rather good effect on the end-to-end delay. In AntHocNet, the end-to-end delay increases as the mobility

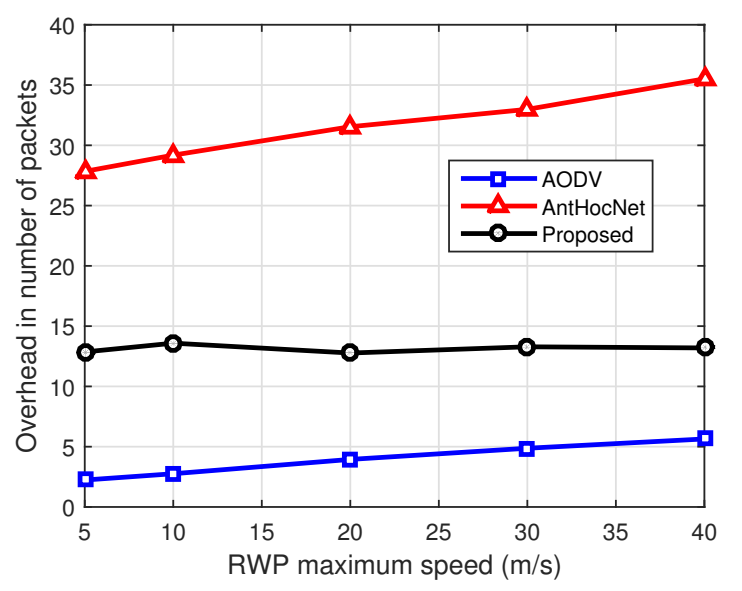

Figure 5: Overhead in number of packets vs. RWP maximum speed.

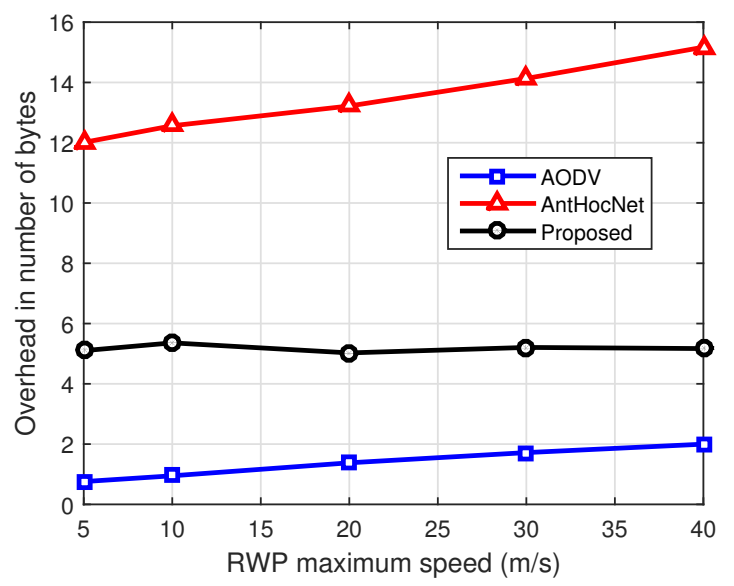

Figure 6: Overhead in number of bytes vs. RWP maximum speed.

increases because it does not adaptively use the shortest path according to the topology changes. On the other hand, the proposed routing protocol shows a gradually decreasing delay performance as the mobility increases because it adaptively uses an alternative path, which provides a smaller routing cost than the previous routing path according to the topology changes.

Figures 5 and 6 show the overhead of the generated control packets versus the maximum node speed for RWP mobility. The overhead in number of packets indicates the total number of control packets transmitted by the nodes of the network versus the data packets delivered to their destination. The overhead in number of bytes indicates the total number of control bytes transmitted versus the data bytes delivered. While both of these are closely related, the overhead in number of packets is important for measuring the MAC efficiency, and the overhead in number of bytes is important for measuring the channel occupancy and the energy consumption of nodes [14]. In AntHocNet, all the nodes in the network periodically transmit hello messages for pheromone 
diffusion, and thus, the overhead is significantly high. On the other hand, AODV has no periodically generated control packets, and thus, its overhead is very low. As the mobility increases, the overheads of both AODV and AntHocNet increase because link failures occur more frequently. However, the overhead of the proposed routing protocol is located between that of AODV and that of AntHocNet and is notably maintained at an almost constant value with respect to the mobility level because a link failure rarely occurs even when the mobility increases.

\section{CONCLUSION}

The proposed bio-inspired routing protocol allows nodes to use the overhearing function, and hence, the one-hop neighbor nodes around the shortest path between the source and the destination are determined autonomously. Then, a pheromone is diffused within only the coverage of one-hop neighbors, and consequently, the overhead of control packet transmission is significantly decreased. Moreover, the proposed routing protocol performs a probabilistic path exploration on the basis of the diffused pheromone and ensures effective alternative paths between the source and the destination. For the route maintenance, two types of ant packets are designed. The exploitation ants serve to secure the shortest path between the source and the destination, and the exploration ants are used for providing the alternative paths around it in order to prepare for link failures. These separate roles of the ants enable the use of the shortest path to the maximum possible extent in the dynamic topology and an effective discovery of alternative paths without the route stagnation problem. The OPNET simulation results show that the proposed routing protocol outperforms the AODV and AntHocNet protocols in terms of the data delivery ratio and the end-to-end delay, and significantly decreases the routing overhead against AntHocNet. The proposed routing protocol shows a higher performance gain as the mobility increases, so it is expected to be applied to MANETs with high mobility and dynamic channels, such as tactical and vehicular ad hoc networks.

\section{ACKNOWLEDGMENTS}

This research was supported by Agency for Defense Development (ADD-IBR-245).

\section{REFERENCES}

[1] Hosseini, S. H., Ghazani, N., Lotf, J. J., and Alg, R. M. 2009. A new survey of routing algorithms in ad hoc networks. In Proceedings of the 3rd International Conference on Communications and information technology (CIT'09), Wisconsin, USA, 296-300.

[2] Marina, M. K. and Das, S. R. 2005. Routing in Mobile Ad Hoc Networks. Ad Hoc Networks, Springer (2005), 63-90.

[3] Hanzo, L. and Tafazolli, R. 2007. A survey of QoS routing solutions for mobile ad hoc networks. IEEE Communications Surveys \& Tutorials. 9, 2 (July 2007), 50-70.

[4] Dressler, F. and Akan, O.B. 2010. Bio-inspired networking: from theory to practice. IEEE Communications Magazines, 48, 11 (Nov. 2010), 176-183.
[5] Bitam, S., Mellouk, A., and Zeadally, S. 2015. Bio-Inspired routing algorithms survey for vehicular ad hoc networks. IEEE Communications Surveys 86 Tutorials, 17, 2 (May 2015), 843-867.

[6] Zhang, Z., Long, K., Wang, J., and Dressler, F. 2014. On swarm intelligence inspired self-organized networking: its bionic mechanisms, designing principles and optimization approaches. IEEE Communications Surveys \& Tutorials, 16, 1 (July 2014), 513-537.

[7] Bonabeau, E. and Theraulaz, G. 2000. Swarm smarts. Scientific American, 282, 3 (2003), 72-79.

[8] Jackson, D. E. and Ratnieks, F. L. 2006. Communication in ants. Current biology, 16, 15 (2006), R570-R574.

[9] Perkins, C. E. and Royer, E. M. 1999. Ad-hoc on-demand distance vector routing. In Proceedings of the 2nd IEEE Workshop on Mobile Computing Systems and Applications, (Feb. 25-26, 1999), New Orleans, LA, 90-100.

[10] Perkins, C. E. 2003. Ad hoc On-Demand Distance Vector ( $A O D V$ ) Routing. IETF RFC 3561.

[11] Perkins, C. and Bhagwat, P. 1994. Highly dynamic destination-sequenced distance-vector routing (DSDV) for mobile computers. In Proceedings of $A C M$ SIGCOMM'94, ACM, New York, USA, 234-244.

[12] Di Caro, G., Ducatelle, F., and Gambardella, L. M. 2005. AntHocNet: an adaptive nature-inspired algorithm for routing in mobile ad hoc networks. European Transactions on Telecommunications (ETT), 16, 5 (Sep. 2005), 443-455.

[13] Ducatelle, F., Di Caro, G., and Gambardella, L. M. 2005. Using ant agents to combine reactive and proactive strategies for routing in mobile ad hoc networks. International Journal of Computational Intelligence and Applications (IJCIA), 5, 2 (2005).

[14] Ducatelle, F. 2007. Adaptive routing in ad hoc wireless multi-hop networks. Ph.D. Dissertation. (May 2007) Lugano, Switzerland.

[15] Marina, M. K. and Das, S. R. 2006. Ad hoc on-demand multipath distance vector routing. Wirel. Commun. Mob. Comput. 6 (2006), 969-988.

[16] Zhou, J., Xu, H., Qin, Z., Peng, Y., and Lei C. 2013 Ad hoc on-demand multipath distance vector routing protocol based on node state. Communications and Network, 5 (2013), 408-413.

[17] Jaisankar, N., and Saravanan, R. 2010. An extended AODV protocol for multipath routing in MANETs. IACSIT International Journal of Engineering and Technology, 2, 4 (2010), 394-400.

[18] Chang, X. 1999. Network simulations with OPNET. In Proceedings of the 31st conference on Winter simulation: Simulation - a bridge to the future-Volume 1, ACM. (Dec. 1999), 307-314.

[19] Choi, H.-H., Lee, H.-G., and Lee, J.-R. 2014. A new energy-aware source routing protocol for maximization of network lifetime in MANET. IEICE Trans. on Information and Systems, E97-D, 2 (Feb. 2014), 335-339.

[20] Heo, W. and Oh, M. 2008. Performance of expanding ring search scheme in AODV routing algorithm. In Proceedings of FGCN '08, (Dec. 13-15, 2008), Hainan Island, 128-132. 